\title{
Natural history of early gastric cancer: a non-concurrent, long term, follow up study
}

\author{
H Tsukuma, A Oshima, H Narahara, T Morii
}

Department of Cancer Control and Statistics, Osaka Medical Center for Cancer and Cardiovascular Diseases, Osaka, Japan H Tsukuma

A Oshima

Department of

Gastroenterology, Osaka Medical Center for Cancer and Cardiovascular Diseases, Osaka, Japan H Narahara

Osaka Cancer

Detection and

Prevention Center,

Osaka, Japan

T Morii

Correspondence to: Dr H Tsukuma, Department of Cancer Control and Statistics, Osaka Medical Center for Cancer and Cardiovascular Diseases, 3-3

Nakamichi 1-chome,

Higashinari-ku, Osaka

537-8511, Japan.

xtukuma@iph.pref.osaka.jp

Accepted for publication 22 June 2000

\begin{abstract}
Background-Controversy has arisen on the natural history of early gastric cancer (EGC). While some emphasise the effectiveness of early detection in reducing mortality from gastric cancer, others insist that EGC is a pseudo-cancer.

Aims/patients/methods-To elucidate the natural history of EGC, a nonconcurrent, long term, follow up study was conducted in 71 patients who were diagnosed endoscopically as having EGC, which was confirmed as cancer on biopsy, but in whom surgical resection was not conducted or delayed by more than six months.
\end{abstract}

Results-The natural course of EGC was observed in 56 cases. Over a period of 6-137 months, 20 remained in the early stage while 36 progressed to the advanced stage. The proportion remaining in the early stage consistently decreased with time. Median duration of those who remained in the early stage was estimated as 44 months. The cumulative five year risk for progressing to the advanced stage was $63.0 \%$. In 38 cases there was no evidence for undergoing surgical resection for gastric cancer. The cumulative five year corrected survival was estimated as $\mathbf{6 2 . 8 \%}$ among those unresected. Hazard rate ratio for gastric cancer mortality was $0.65(p=0.34)$ for screening detected versus non-screening detected. Hazard rate ratio for gastric cancer mortality was 0.51 , significantly lower for patients whose operations were delayed compared with those unresected.

Conclusions-Although EGC showed a relatively long natural history in general, it progressed to the advanced stage with time and led to death from gastric cancer for the most part if left untreated. (Gut 2000;47:618-621)

Keywords: early gastric cancer; natural history

Gastric cancer is still one of the major causes of cancer deaths, ${ }^{1}$ although mortality has been consistently decreasing. ${ }^{2}$ Early detection and early treatment are believed to be effective in reducing mortality from gastric cancer, especially in Japan, ${ }^{34}$ where the incidence of this disease is the highest in the world. ${ }^{5}$ In 1983, we presented data $^{6}$ on the natural course of 43 early gastric cancer (EGC) cases and survival for 34 untreated EGC patients; we suggested that early diagnosis and early treatment may lead to a reduction in mortality from gastric cancer. The study comprised 56 cases who were diagnosed endoscopically as having EGC before 1976 but on whom surgical resection was not conducted or delayed for more than six months. These cases were followed up until November 1980. Recently, however, controversy has arisen on the natural history of EGC, implying that EGC is a pseudo-cancer which rarely progresses to an advanced stage. ${ }^{78}$ To examine the natural history of EGC more clearly, a non-concurrent, long term, follow up study was conducted in EGC patients on whom surgical resection was not conducted or delayed by more than six months after diagnosis. Special attention was paid to the study design so that sufficiently long follow up periods were studied.

\section{Methods}

STUDY SUBJECTS

Eligibility of study subjects was essentially the same as in the previous report in $1983^{6}$ : patients who were diagnosed endoscopically as having EGC, confirmed as cancer on biopsy, but in whom surgical or endoscopic resection was not conducted or delayed by more than six months (fig 1). To ensure long follow up periods of more than 10 years, the study was restricted to patients diagnosed before the end of October 1988 and followed up until the end of October 1998. Based on the hospital cancer registry of Osaka Medical Center for Cancer and Cardiovascular Disease (OMCC) and the screening detected EGC patients' files kept at the Osaka Cancer Detection and Prevention Center (OCDPC), candidates for the study were systematically retrieved. These candidates were collated with the database of the Osaka cancer registry, ${ }^{5}$ a population based cancer registry covering all of Osaka prefecture since December 1962. A questionnaire was mailed to referred medical doctors or patients' families, where possible, to confirm if they had undergone surgical resection or follow up examinations for gastric cancer. In addition to the 56 cases of the previous study in 1983, 15 patients were found to meet the above mentioned criteria. A total of 71 cases were eligible for this study. Vital status as of the end of October 1998 was assessed by referral to residential registrations of municipality offices. Causes of death were confirmed by death certificate.

Abbreviations used in this paper: EGC, early gastric cancer; OMCC, Osaka Medical Center for Cancer and Cardiovascular Disease; OCDPC, Osaka Cancer Detection and Prevention Center. 
Eligibility of the study subjects:

Patients diagnosed endoscopically as having EGC and confirmed as cancer with biopsy, but on whom surgical or endoscopic resection was not conducted or delayed more than six months. Their diagnosis was done before October 1988.

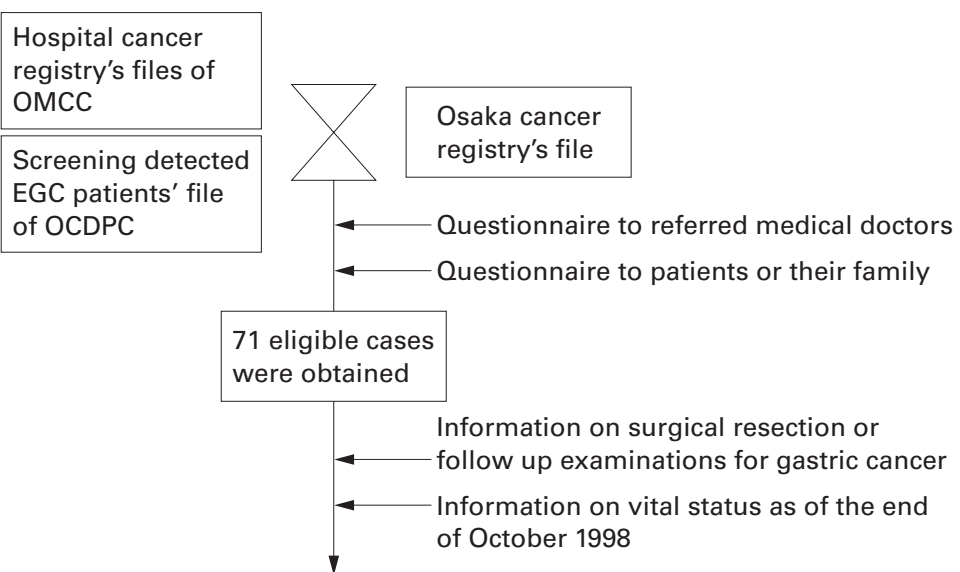

Study subjects and their outcome

- Natural courses of EGC were elucidated for 56 cases: 20 cases remained in the early stage

36 cases progressed to the advanced stage

- Survival analysis:

38 cases had never undergone surgical resection 35 died, 1 alive, 2 unknown for vital status

33 cases had undergone delayed surgical resection 26 died, 7 unknown for vital status

Figure 1 Sources of the study subjects and information, together with outcome of follow up.

STATISTICAL ANALYSES

The duration of the early stage and cumulative corrected survival were estimated using the Kaplan-Meier method and differences between screening detected and non-screening detected were analysed using the Cox proportional hazards model after adjustment for sex and age at diagnosis. Cases who died from causes other than gastric cancer were regarded as censored. Patients with unknown vital status were also treated as censored at the time when they were last known to be alive. Survival was compared between unresected patients and those whose operations were delayed. The log rank test was used for statistics on the difference between the two survival curves. $p$ values less than 0.05 for both tailed were considered statistically significant. The statistical package STATA ${ }^{9}$ was used for analyses.

\section{Results}

For 56 of the total 71 study subjects, the natural course of EGC was elucidated through subsequent examinations with endoscopy and/or double contrast $x$ ray, or pathological findings on postoperative material (fig 1). Over a period of 6-137 months (mean 39 months), 20 remained in the early stage while 36 progressed to the advanced stage. Figure 2 shows KaplanMeier estimates of the proportion remaining in the early stage. The proportion remaining in the early stage decreased consistently with time. Median duration of those who remained in the early stage was estimated as 44 months. Cumulative five year risk for progressing to the advanced stage was estimated as $63.0 \%$ (95\%

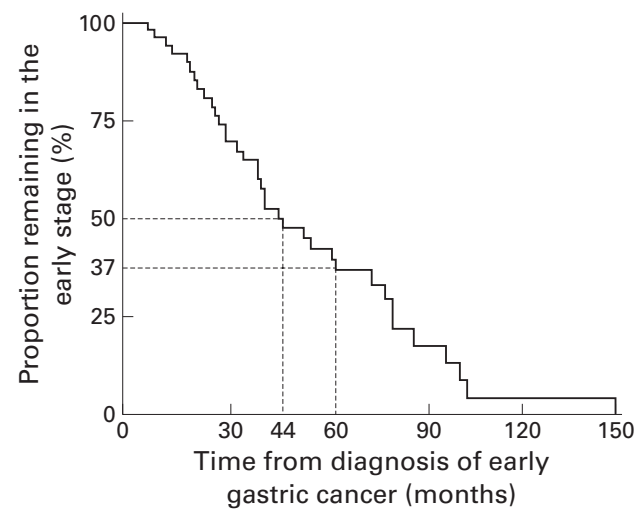

Figure 2 Kaplan-Meier estimates of proportion of cases who remained in the early stage of gastric cancer.

confidence interval (CI) 48.1-77.9\%). Among the 56 cases, 27 were detected through screening programmes for gastric cancer while the remaining 29 were diagnosed at outpatient clinics by various symptoms. Age and sex adjusted hazard rate ratio for progressing to the advanced stage was 0.90 (95\% CI $0.45-1.79$; $\mathrm{p}=0.77)$ in the screening detected compared with the non-screening detected cases.

In 38 cases there was no evidence of surgical resection for gastric cancer, although in two of these cases vital status as of the end of October 1998 was unknown. During the follow up period of 13-207 months (mean 72 months), 35 cases had died while one patient was alive (fig 1). Twenty three died from gastric cancer while nine died from other causes. Cause of death was unknown for the remaining three cases. When these three cases were regarded as gastric cancer deaths, cumulative five year corrected survival of the 38 unresected cases was estimated as $62.8 \%$ (95\% CI $46.6-79.0 \%)$. If these three were regarded as censored, the corrected survival rate was estimated as $67.8 \%$. Among the 38 unresected cases, 18 were detected through screening programmes for gastric cancer while the remaining 20 were diagnosed at outpatient clinics by symptoms. When the three cases of unknown cause of death were regarded as gastric cancer deaths, the hazard rate ratio for gastric cancer mortality was 0.65 (95\% CI $0.28-1.55$; $\mathrm{p}=0.34$ ) in the screening detected compared with the outpatient detected cases.

Concerning the 33 cases whose operations were delayed, 19 gastric cancer deaths and four deaths from other causes were observed, as well as three unknown causes of death, over a period of 10-213 months (mean 104 months). Vital status of the remaining seven cases was unknown as of the end of October 1998, although their living status had been confirmed 73-183 months (mean 122 months) after diagnosis of EGC (fig 1). The periods between diagnosis of EGC and surgical operation were 6-12 months in seven patients, 13-24 months in eight, $25-36$ months in six, 37-48 months in two, 49-60 months in five, and more than 60 months in five. Postoperative findings of the lesions revealed advanced gastric cancer in 18 cases and early gastric cancer in 13. The remaining two were unknown. Their cumula- 


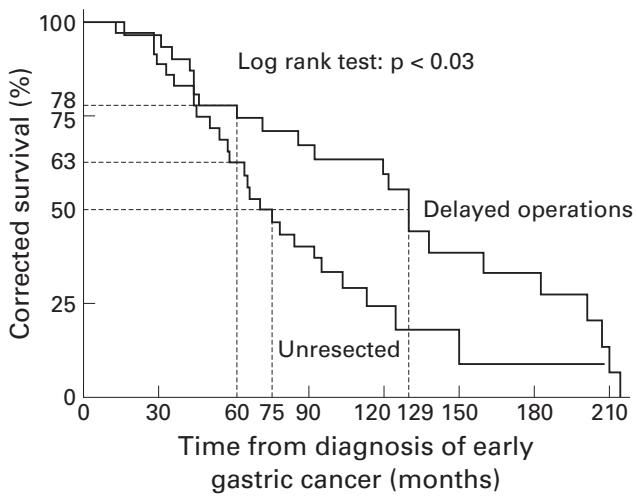

Figure 3 Kaplan-Meier estimates for corrected survival in unresected cases and in those whose operations were delayed.

tive five year corrected survival rate was estimated as $78.1 \%$ (95\% CI $63.7-92.3 \%$ ) when the three unknown causes of death were regarded as gastric cancer death. If these three cases were treated as censored, the corresponding value was $78.1 \%$, the same as the former assumption.

Figure 3 shows Kaplan-Meier estimates for corrected survival in unresected cases and in patients whose operations were delayed, together with median survival. The corrected survival curves were significantly different (log rank test: $\mathrm{p}<0.030$ ). Age and sex adjusted hazard rate ratio for gastric cancer mortality was 0.51 (95\% CI $0.27-0.96$; $\mathrm{p}=0.039$ ), significantly lower in cases whose operations were delayed compared with those unresected.

\section{Discussion}

As five year relative survival of postoperative patients with EGC was reported to reach $90 \%$ or more, ${ }^{10}{ }^{11}$ our results strongly suggest that EGC, if left untreated, would progress to the advanced stage and lead to death from gastric cancer with time. There was no evidence to suggest that screening detected EGC would not progress to the advanced stage and rarely lead to death from gastric cancer. Although these findings were shown in the previous study, they were confirmed here in this study with a follow up period of more than 10 years. Thus we believe that EGC is not a pseudocancer although it shows a relatively long natural history in general. Patients who are diagnosed as having EGC should be treated appropriately as early as possible.

Some limitations inherent in this study should be discussed. Firstly, diagnosis of EGC was based on endoscopic and not pathological findings. As EGC is defined as adenocarcinoma where cancer invasion remains in the mucosal or submucosal layers, pathological confirmation is necessary for accurate diagnosis. Our study subjects who were diagnosed as having progressed to the advanced cancer, therefore, might have been in the advanced stage from the start of the observation. However, diagnostic accuracy of EGC with endoscopy was reported to be $80 \%$ or more. ${ }^{12}$ It is unlikely that all cases diagnosed as having progressed to the advanced stage at subsequent examinations had been in the advanced stage from the start of the study.

Secondly, subjects were not a representative sample of EGC patients but seemed to be older (mean age at diagnosis 64.1 years (range 34-84)). Crude mortality rate, therefore, would be higher among the study subjects than among all EGC cases. As survival analysis focused on mortality from gastric cancer in this study, this type of bias would have had a competing risk. That is, the study subjects might have died from other causes before death from gastric cancer. We consider that the effect on gastric cancer mortality in this study would have been underestimated.

Thirdly, the results on the natural course of EGC may have been biased as they were observed only for 56 of the original 71 cases. Characteristics possibly relating to cancer progression were compared between the 56 cases and the remaining 16 cases. The proportion in the screening detected group was $48.2 \%$ among the former and $60.0 \%$ in the latter. The proportion of those with well or moderately differentiated adenocarcinoma was $59.6 \%$ and $63.6 \%$, respectively. These findings suggest that the results obtained may have been biased towards rapid growing cases, but the magnitude of this bias does not appear to be important. Cause specific survival was further compared between the two groups using Cox regression analysis in which variables such as age, sex, history of gastrectomy, macroscopic type of EGC, and histology were included. The multivariate adjusted hazard rate ratio was 0.77 for the 56 cases, but not statistically different from 1.0 .

It has recently been reported that diagnostic criteria for gastric cancer are different between Japanese and Western pathologists, in particular for intestinal type EGC. ${ }^{13}$ The Japanese tended to diagnose mucosal gastric cancer even if there was no evidence of invasion into the lamina propria whereas Western pathologists never diagnosed cancer without invasion of the lamina propria. If it were applicable to the present study subjects, some borderline lesions of such adenomas and dyplasias, as might be termed by Western pathologists, have been included. Subjects with well or moderately differentiated adenocarcinoma showed lower hazard rate ratios for progression to the advanced stage (0.68 and 0.45 , respectively) compared with those with undifferentiated or poorly differentiated adenocarcinoma but the differences were not significant. Furthermore, the study included a total of 36 cases that were detected through screening programmes for gastric cancer. Therefore, it is likely that the study subjects may have included rather slow growing cases in relation to length biased sampling or over diagnosis, ${ }^{14}$ as indicated in this study (hazard rate ratio for gastric cancer mortality was 0.65 in the screening detected cases). Despite these possibilities of misclassifications and biases, the results showed that EGC, if left untreated, would progress to the advanced stage and lead to death from gastric cancer.

Duration of EGC and survival of unresected EGC cases showed essentially the same results 
as our former study. ${ }^{6}$ In the present study, we attempted to examine the effect of delayed operation on mortality from gastric cancer because it was important to clarify if advocating surgical resection would still benefit the patient. The results suggested that EGC patients would still benefit even if surgery was delayed for more than six months after diagnosis. However, we would urge caution in this interpretation as those individuals who were in the delayed operation group had to survive long enough to have surgery. Thus this may have led to an overestimate of survival in the delayed surgery group. However, this is unlikely to have had a major effect on the results as few patients died quickly, and follow up was long compared with the time delay before surgery.

At present, there are several observational studies supporting the effectiveness of early detection and early treatment in reducing mortality from gastric cancer ${ }^{3}$ while others have argued against it. ${ }^{78}$ In their report, Everett and Axon $^{7}$ claimed that EGC is a pseudo-disease, possibly based on the results of the study of von Holstein and colleagues. ${ }^{8}$ von Holstein and colleagues $^{8}$ compared 354 postgastrectomy patients who accepted endoscopic screening with 484 who either declined or were not offered investigation. Over 17 years of follow up, 17 EGCs were detected in the screening group compared with two cases in the control group. The number of deaths from gastric cancer in the two groups was similar (12 $v 14)$. The authors suggest that screening detected EGC is a pseudo-cancer. However, the study did not use random allocation, and thus it was possible that some bias affected the results. As the study subjects were all postgastrectomy patients, there is no guarantee that the study results are applicable to the general population. von Holstein et al commented only on the significance of regular endoscopic screening for postgastrectomy patients.

Our long term follow up study, together with previously reported case control studies ${ }^{14}{ }^{15}$ on the effectiveness of screening programmes, strongly suggest that early detection and early treatment are effective in reducing mortality from gastric cancer. To confirm this, however, it is necessary to conduct a randomised controlled trial of endoscopic screening of the general population. ${ }^{16}$ Endoscopic mucosal resection for gastric cancer has recently been developed. It is known to be safe and equivalent to surgical resection. In combining the present evidence, we conclude that resection, including endoscopic mucosal resection in cases of mucosal cancer, is the first choice for those patients who are diagnosed as having EGC through screening programmes.

We thank the Osaka cancer registry, the hospital cancer registry of OMCC, and the OCPDC for use of their databases, and Sachiko Endoh, Mayumi Miyamoto, and Yoko Kinoshita for obtaining information on study subjects and their prognosis. This study was supported in part by a grant for New 10-year Strategy for Cancer Control, Prevention of Cancer, from the Japanese Ministry of Health and Welfare.

1 Pisani P, Parkin DM, Ferlay J. Estimates of the worldwide mortality from twenty-five major cancers in 1990. Int $\mathcal{F}$ Cancer 1999;83:18-29.

2 Tominaga S, Kuroishi T, Aoki K. Cancer mortality statistics in 33 countries 1953-1992. UICC, Nagoya: Roppo Shuppan Co. Ltd, 1998.

3 Oshima A. Screening for stomach cancer: the Japanese program. In: Chamberlan J, Miller AB, eds. Screening for gastrointestinal cancer.

4 Hisamichi S. Screening for gastric cancer. World f Surg 1989:13:31-7.

5 Parkin DM, Whelan SL, Ferlay J, et al eds. Cancer incidence in five continents, vol. vii. IARC Scientific Publication No143. Lyon: IARC, 1997.

6 Tsukuma H, Mishima T, Oshima A. Prospective study of "early" gastric cancer. Int f Cancer 1983;31:421-6.

7 Everett SM, Axon ATR. Early gastric cancer: disease or pseudo-disease? Lancet 1998;351:1350-2.

8 Stael von Holstein C, Eriksson S, Huldt B, et al. Endoscopic screening during 17 years for gastric stump carcinoma. A prospective clinical trial. Scand f Gastroenterol 1991;26: 1021-6.

9 STATA Reference Manual Release 2. Santa Monica: Computing Resource Center, 1990.

10 Miwa K. Cancer of the stomach in Japan. In: Kajitani T, Koyama Y, Umegaki Y, eds. Recent results of cancer treatment in Japan. Gann Monograph on Cancer Research, No 22. in Fapan. Gann Monograph on Cancer Research, No

11 Sue-Ling HM, Martin I, Griffith J, et al. Early gastric Sue-Ling HM, Martin I, Griffith J, et al. Early gastric 1318-22.

12 Okuda S. Differential diagnosis of early gastric cancer from advanced carcinoma. In: Murakami T, ed. Early gastric cancer. Gann Monograph on Cancer Research, No 11. Tokyo: University of Tokyo Press, 1971:283-301.

13 Schlemper RJ, Itabashi M, Kato Yo, et al. Differences in diagnostic criteria for gastric carcinoma between Japanese and Western pathologists. Lancet 1997;349:1725-9.

14 Oshima A, Hirata N, Ubukata T, et al. Evaluation of mass screening program for stomach cancer with a case-control study design. Int $\mathcal{F}$ Cancer 1986;38:829-33.

15 Fukao A, Tsubono Y, Tsuji I, et al. The evaluation of screening for gastric cancer in Miyagi Prefecture, Japan: a population based case-control study. Int f Cancer 1995;60: popula 8 .

16 Cole P, Morrison AS. Basic issues in cancer screening. In: Screening in Cancer, UICC Technical Report Series, vol. 40. Geneva: UICC, 1978;7-39. 